Résumés des conférences et travaux

\title{
Manuscrits hébreux et judéo-arabes médiévaux
}

Conférences de l'année 2013-2014

\section{Judith Olszowy-Schlanger}

\section{OpenEdition \\ Journals}

\section{Édition électronique}

URL : https://journals.openedition.org/ashp/1679

DOI : 10.4000/ashp.1679

ISSN : 1969-6310

\section{Éditeur}

Publications de l'École Pratique des Hautes Études

Édition imprimée

Date de publication : 1 septembre 2015

Pagination : 14-20

ISSN : 0766-0677

Référence électronique

Judith Olszowy-Schlanger, "Manuscrits hébreux et judéo-arabes médiévaux », Annuaire de l'École pratique des hautes études (EPHE), Section des sciences historiques et philologiques [En ligne], 146 | 2015 mis en ligne le 28 septembre 2015, consulté le 07 juillet 2021. URL : http://journals.openedition.org/ ashp/1679; DOl : https://doi.org/10.4000/ashp.1679 


\title{
MANUSCRITS HÉBREUX ET JUDÉO-ARABES MÉDIÉVAUX
}

\author{
Directeur d'études : M ${ }^{\text {me }}$ Judith OLSzOWy-Schlanger
}

Programme de l'année 2013-2014: Typologie des écritures hébraïques I : écritures carrée orientales du IXe au XII e siècle.

Nos séminaires de recherche de cette année ont été consacrés à la paléographie, et plus particulièrement à la typologie des écritures hébraïques. Depuis l'établissement de la paléographie hébraïque comme une discipline universitaire à part entière, à l'EPHE et à l'IRHT (CNRS), par Colette Sirat, et à la Bibliothèque nationale et universitaire d'Israël, par Malachi Beit-Arié, les efforts du projet franco-israélien se sont concentrés d'une part sur l'élaboration d'outils méthodologiques indispensables (méthodologie de recherche, recension de manuscrits datés), et d'autre part sur l'étude des aspects codicologues quantifiables des manuscrits datés qui ont abouti à une base de données Sfardata et à la publications des facsimilés des manuscrits datés ${ }^{1}$. Les travaux systématiques du projet de paléographie hébraïque ont posé des bases pour l'étude de la typologie des écritures, en distinguant des grands types d'écritures selon des zones géo-culturelles : écriture orientale, ashkénaze, sépharade, italienne et byzantine. En partant de ces grandes lignes tracées par ces travaux pionniers, nous pouvons aujourd'hui travailler sur la typologie de manière plus précise, en essayant de dégager des sous-groupes à l'intérieur de ces grandes zones géo-culturelles. En effet, une typologie plus fine permettant de différencier les écritures locales ou limitées à une période au sein d'une même grande entité n'est qu'à ses débuts.

Nos séminaires de cette année avaient pour but l'étude des sous-types d'écritures hébraïques, avec une attention particulière à la typologie plus fine à l'intérieur du groupe oriental. Nous avons étudié un corpus sélectionné de manuscrits et documents datés et datables du $\mathrm{IX}^{\mathrm{e}}$ au XI ${ }^{\mathrm{e}}$ siècles, en nous concentrant sur les documents provenant d'Égypte, de Palestine, d'Iraq et d'Afrique du Nord. Ce corpus, qui contient les plus anciens manuscrits médiévaux connus, provient principalement de la Geniza du Caire ainsi que des collections constituées au XIX ${ }^{\mathrm{e}}$ siècle par un savant caraïte, Abraham Firkovicz, lors de ses voyages en Égypte et au Proche-Orient, et conservées aujourd'hui à la Bibliothèque nationale de Russie à Saint-Pétersbourg. Une étude préliminaire des aspects paléographiques des documents de la Geniza du Caire a été publiée par Edna Engel ${ }^{2}$. Notre recherche s'inspire de cette publication mais arrive parfois à des conclusions qui diffèrent légèrement.

Le corpus étudié est composé de manuscrits copiés principalement en Égypte - à Fustat mais aussi dans d'autres villes et villages égyptiens, en Babylonie (Iran et Iraq)

1. http://sfardata.nli.org.il

2. E. Engel, «Style of the Hebrew script in the tenth and eleventh centuries in the light of dated and datable Genizah documents » (en hébreu), Te`uda, 15 (1998). 
ainsi qu'en Afrique du Nord (principalement à Kairouan). Le rôle de Fustat comme un centre d'échanges commerciaux et intellectuels important explique pourquoi les manuscrits produits dans ces endroits différents se sont retrouvés déposés dans la Geniza de la synagogue Ben Ezra. L'attrait exercé par cette ville explique aussi la présence des scribes d'origines différentes et la coexistence des écritures typologiquement variées. On constate également une très grande diversité d'écritures personnelles, ce qui rend la typologie difficile. Il convient aussi de prendre en considération les styles (genera scribendi) d'écriture différents au sein d'une même entité typologique (écriture dite carrée et écritures documentaires plus cursives), ainsi que la qualité variable (calligraphique, soignée, informelle, peu entraînée, etc.) avant d'attribuer un écrit à un type ou sous-type.

Malgré ces mises en gardes méthodologiques, il est possible de distinguer clairement, à l'époque qui nous concerne, deux sous-groupes au sein de l'écriture orientale que nous allons désigner par des termes géographiques : 1. le groupe du Sud-Ouest, 2. le groupe du Nord-Est. L'existence des deux sous-types d'écriture carrée orientale a été observée par Edna Engel, qui les a placées dans une séquence chronologique : écriture proto-carrée (correspondant à notre groupe du Sud-Ouest) et carrée orientale (notre groupe du Nord-Est) ${ }^{1}$. Nous préférons le terme qui implique la distinction géographique plutôt que chronologique. En effet, si certaines caractéristiques de la «protocarrée » se trouvent dans l'écriture hébraïque de l'époque byzantine (par exemple, les fragments du livre des Rois, Oxford, Ashmolean Museum, Sackler Library, Antinoopolis Papyri $\mathrm{n}^{\text {os }} 47$ et 48 , trouvés par les archéologues en 1912 avec des documents coptes datable du $\mathrm{V}^{\mathrm{e}}$ au $\mathrm{IX}^{\mathrm{e}}$ siècle $^{2}$ ), les manuscrits médiévaux conservés montrent que les deux écritures continuaient à être employées en parallèle, à la même époque mais dans des milieux différents. La distinction entre l'écriture " irakienne » et l'écriture « de l'Ouest » se trouve d'ailleurs dans des textes médiévaux, tels que l'inventaire des livres de 1080 de la synagogue de la congrégation de rite babylonien à Fustat (TS 20. $47)^{3}$.

La division fondée sur l'origine géographique présumée de ces sous-types de l'écriture orientale n'implique pas toujours le lieu de production des manuscrits. À l'époque dite «classique » de la Geniza (époque Fatimide), les scribes formés dans les deux grandes traditions pouvaient se déplacer (par exemple, les migrations bien attestées aux $\mathrm{IX}^{\mathrm{e}}-\mathrm{X}^{\mathrm{e}}$ siècles de l'Iraq abbaside vers l'Égypte et l'Afrique du Nord) et se trouver notamment en Égypte. De plus, les sous-types d'écriture correspondaient aussi aux centres intellectuels différents même s'ils étaient situés dans la même ville. Il sera notamment possible, pour la période ancienne qui nous intéresse ici, de différencier entre les sous-types d'écriture qui correspondent respectivement au rituel (et les institutions communautaires et éducation des scribes) «palestinien » et « babylonien ». Ainsi, sans toujours mener à une indication fiable du lieu de production d'un

1. E. Engel, « Style of the Hebrew script in the tenth and eleventh centuries in the light of dated and datable Genizah documents » (en hébreu), Te ‘uda, 15 (1998), p. 369-371.

2. C. Sirat, Les papyrus en caractères hébraïques trouvés en Égypte, Paris, édition du CNRS, 1985, p. 3537.

3. N. Allony (éd. M. Frenkel, H. Ben-Shammai, with the participation of M. Sokolow), The Jewish Library in the Middle Ages. Book Lists from the Cairo Genizah (en hébreu), Jérusalem, 2006, nº 80, p. 296. 
manuscrit ou document, l'identification du sous-type de l'écriture peut en revanche indiquer l'origine du scribe ou le milieu religieux et intellectuel auquel il appartenait. Voici quelques traits pertinents des deux sous-groupes d'écriture carrée ${ }^{1}$.

\section{Écritures carrées}

L'écriture orientale du Sud-Ouest est un type d'écriture hébraïque employé principalement en Palestine et en Égypte, qui depuis l'époque byzantine constituaient le même milieu culturel quant aux communautés et traditions juives. Il y a très peu d'écrits explicitement datés et localisés dans ce type d'écriture. Mais on constate que ce sous-type d'écriture est employé dans les écrits dont le contenu ou l'histoire sont liés à l'aire palestino-égyptienne et au rite juif palestinien correspondant. Cette écriture est présente dans des fragments considérés comme appartenant aux couches les plus anciennes de la Geniza du Caire tels que les palimpsestes. Elle a continué à être employée jusqu'au $\mathrm{XI}^{\mathrm{e}}$ siècle.

Le corpus d'écrits dans cette écriture inclut :

a) les palimpsestes de la Geniza du Caire où le texte supérieur en caractères hébraïques est copié sur des textes monastiques recyclés en grec, araméen chrétien palestinien, arménien, géorgien ou hébreu ${ }^{2}$;

b) les fragments des textes bibliques dont certains sont pourvus de voyelles selon la tradition palestinienne, d'autres proviennent des Pentateuques qui contiennent des marques de subdivision du texte en portion de lecture hebdomadaire selon le cycle de trois ans (sedarim) pratiqué dans les synagogues suivant le rite palestinien;

c) les textes liés au rite ou aux institutions palestiniennes tels que le Talmud de Jérusalem, le Targum palestinien, les poèmes liturgiques.

Les traits caractéristiques pertinents de ce sous-type d'écriture orientale, définis très brièvement ici incluent (tableau 1$)^{3}$ :

1. Pour une étude récente des écritures documentaires plus cursives, voir J. Olszowy-Schlanger, « Early Babylonian "documentary" script: diplomatic and palaeographical study of two Geonic letters from the British Library Genizah Collection », dans N. de Lange, J. Olszowy-Schlanger (éd.), Manuscrits hébreux et arabes. Mélanges en l'honneur de Colette Sirat, Turnhout, Brepols, 2014 (Bibliologia 38), p. 177-195.

2. Voir M. Sokoloff et J. Yahalom, « Christian palimpsests from the Cairo Geniza », Revue d'histoire des textes, 8 (1978), p. 109-132 ; N. Tchernetska, «Greek Oriental palimpsests in Cambridge: problems and prospects ", dans C. Holmes, J. Waring (éd.), Literacy, Education and Manuscript Transmission in Byzantium and Beyond, Leyde - Boston - Cologne, Brill, 2002 ; N. Tchernetska, J. Olszowy-Schlanger, N. de Lange, «An early Hebrew-Greek biblical glossary from the Cairo Geniza », Revue des études juives, 166 (2007), p. 91-128 ; J. Olszowy-Schlanger, « An early palimpsest scroll of the Book of Kings from the Cairo Geniza », dans B. M. Outhwaite, S. Bhayro (éd.), "From a Sacred Source”. Genizah Studies in Honour of Professor Stefan C. Reif, Leyde - Boston, Brill, 2010, p. 237-247 ; J. OlszowySchlanger, Roni Shweka, « Newly discovered early palimpsest fragments of the Talmud Yerushalmi from the Cairo Genizah », Revue des études juives, 172 (2013), p. 49-81 ; J. Olszowy-Schlanger, « On the Hebrew script of the Greek-Hebrew palimpsests from the Cairo Genizah », dans The Jewish-Greek Tradition in Antiquity and the Byzantine Empire, Cambridge, CUP, 2014, p. 279-299.

3. Pour une discussion détaillée des trais pertinents de l'écriture hébraïque orientale du Sud-Ouest dans quelques palimpsestes de la Geniza du Caire, voir J. Olszowy-Schlanger, « Hebrew script of the GreekHebrew palimpsests », p. 295. 
a) les bases horizontales des lettres beth, tet, kaph, mem, nun, 'ayin et pe qui forment un angle de $20-25^{\circ}$ par rapport à la ligne de base, ce qui donne à la ligne d'écriture un aspect penché vers la gauche ;

b) aspect général angulaire et pointu, dû à la présence de longs sérifs additionnels, à la finition en pointe des traits verticaux ainsi qu'à la forme en diamant des têtes des lettres gimel, zayin et nun;

c) les traits descendants des lettres kaph, nun, pe, sade finaux et qoph sont très courts, ne dépassant presque pas ou de très peu la ligne de base ;

d) en revanche, des lettres qui sont, dans d'autres types d'écriture, le plus souvent incluses dans la hauteur de la ligne d'écriture et ont la hauteur d'une lettre moyenne, telles que gimel, 'ayin et pe, sont très longues et descendent sous la ligne de base ;

e) d'autres lettres, telles que lamed et resh, sont caractérisées par des traits verticaux très courts qui n'atteignent pas la ligne de base ;

f) certaines lettres ont une forme très distinctive, dont la structure a des caractéristiques communes d'un manuscrit à l'autre, malgré des différences évidentes dues à l'individualité des scribes.

Aleph : sa structure de base est composée de trois traits principaux : le trait central oblique, le trait vertical de gauche et le trait vertical de droite. La spécificité concerne plus particulièrement le trait vertical de gauche. Ce trait est presque perpendiculaire à la ligne de base et relie la ligne de crête avec la ligne de base. Il descend de l'extrémité du trait central oblique, et même le dépasse souvent vers le haut. Il est souvent fini par un pied tourné dans la plupart des cas vers la droite, vers l'intérieur de la lettre.

Pe est particulièrement grand, plus large et plus long qu'une lettre moyenne. Il est composé de quatre traits : une longue barre horizontale presque parallèle à la ligne de crête, un trait vertical de droite presque perpendiculaire à la ligne d'écriture, une longue base plus longue que la barre supérieure et penchée par rapport à la ligne de base et un «nez » - trait de gauche qui est relativement long, courbé vers l'intérieur de la lettre, tracé d'un mouvement séparé, perpendiculaire à la barre horizontale supérieure qu'il dépasse vers le haut.

Comme déjà mentionné, la plupart des manuscrits dans ce type d'écriture ne sont pas datés. Quelques indications sur l'ancienneté de ce type d'écriture peuvent être proposées. Fragment TS NS 308.25 est un fragment d'un codex contenant des textes liturgiques ${ }^{1}$. Sur le côté chair, il y a un modèle de lettre de divorce. Ce modèle contient une date : 4633 AM (=872/873 AD) et le lieu du divorce : Jérusalem. Il s'agit bien d'un formulaire-modèle et non pas d'un document juridique. Il est néanmoins probable que le scribe du codex et du formulaire ait pris un document existant comme modèle du texte de la lettre de divorce. Par conséquent, la date 872 / 873 est un terminus post quem pour ce modèle de lettre de divorce et de son écriture, mais ce modèle n'était probablement pas très éloigné dans le temps et dans le lieu de la lettre de divorce elle-même. Ce type d'écriture est employée au $\mathrm{x}^{\mathrm{e}}$ sièce, comme le montrent quelques

1. Voir R. Brody, A Hand-list of Rabbinic manuscripts in the Cambridge Genizah Collections, Cambridge, CUP, 1998, p. 197. E. M. Margaliot, Hilkhot Ereș Israel min ha-Genizah (en hébreu), Jérusalem, 1973, p. 121. 
fragments des codex bibliques, tels que TS A 39.11+Manchester, John Rylands Library Gaster Geniza $2^{1}$ (fragments conservés proviennent du livre de Jérémie, manuscrits a été copié ou acquis (le colophon est endommagé, mais la forme קניתי, "j'ai acquis 》 plutôt que כתבתי, «j'ai écrit », est probable) en 953/954 à Gaiffa en Égypte et TS A 42.2+TS B 17.38+TS NS 283.123+TS NS 80.14, fragments d'un codex des Haphtarot, copié en 924 en Égypte ou en Palestine car le cycle de lecture liturgique de la Bible indiqué dans le manuscrit est celui du rite palestinien ${ }^{2}$.

L'écriture orientale du Nord-Est semble originaire de la Babylonie de l'époque abbasside, mais cette écriture, souvent très calligraphique, est vite répandue dans d'autres parties du monde de l'Islam. Les exemples datés et localisés de cette écriture proviennent en effet d'Iran (TS AS 62.402, 461, 492-493, 533, 644+TS NS 246.26.2 and 18(a)+TS NS 283.10, copié en 903/4 à Gunbad-i-Mallgàn ${ }^{3}$; "Codex Babylonicus » Saint-Pétersbourg, Firkovicz EBP I B 3, copié en 916, tous les deux avec la vocalisation babylonienne ${ }^{4}$ ) ou d'Égypte ou Palestine. Ce sous-type inclut notamment des codex massorétiques, modèles de grande qualité pourvus de voyelles de type tibérien, et parmi eux le fameux Codex d'Alep qui aurait été copié à Tibériade ${ }^{5}$ ou le Codex de Leningrad (MS Firkovicz, EBP I B 19a, copié par Samuel ben Jacob au Caire en 1008, ainsi que d'autres manuscrits de ce scribe ${ }^{6}$ ), ainsi qu'un grand nombre de manuscrits de la Geniza du Caire du $\mathrm{X}^{\mathrm{e}}$ au XII ${ }^{\mathrm{e}}$ siècle. Ce sous-type a également été employé par les scribes caraïtes dans leurs livres et documents juridiques au $\mathrm{X}^{\mathrm{e}}$ et $\mathrm{XI}^{\mathrm{e}}$ siècle.

Les traits caractéristiques pertinents de ce sous-type d'écriture orientale, définis très brièvement ici incluent (tableau 1$)^{7}$ :

a) les bases horizontales des lettres beth, tet, kaph, mem, nun, 'ayin et pe sont presque parallèles à la ligne de base ;

b) les sérifs additionnels sont courts, souvent limités à l'attaque de plume ou à un petit traits à l'extrémité gauche des barres horizontales supérieures ;

c) les traits descendants des lettres kaph, pe, sade finaux et qoph sont le plus souvent longs (le nun peut être court) ; ils descendent sous la ligne de base, par une longueur de la moitié de la hauteur de la ligne d'écriture ou plus ;

d) les lettres gimel, 'ayin et pe sont de la taille des lettres moyenne; elles sont incluses entre la ligne de crête et la ligne de base;

e) la lettre lamed souvent ne touche pas à la ligne de base, en revanche la lettre resh a la hauteur de la ligne d'écriture ;

1. M. Beit-Arié, C. Sirat, M. Glatzer, Codices Hebraicis Litteris exarati quo tempore scripti fuerint exhibentes, t. I, jusqu'à 1020, Brepols, 1997 (MPMA, nº 9).

2. Beit-Arié, Sirat, Glatzer, Codices, I, 1997, nº 4.

3. Beit-Arié, Sirat, Glatzer, Codices, I, 1997, $n^{\circ} 2$.

4. Beit-Arié, Sirat, Glatzer, Codices, I, 1997, nº 3.

5. Beit-Arié, Sirat, Glatzer, Codices, I, 1997, nº 6 et la bibliographie mentionnée.

6. Beit-Arié, Sirat, Glatzer, Codices, I, 1997, nº 17.

7. Pour une discussion détaillée des trais pertinents de l'écriture hébraïque orientale du Sud-Ouest dans quelques palimpsestes de la Geniza du Caire, voir J. Olszowy-Schlanger, « Hebrew script of the GreekHebrew palimpsests », p. 295. 
f) les lettres distinctives aleph et pe ont un ductus et une morphologies différents du sous-groupe du Sud-Ouest.

Aleph : sa structure de base est composée de trois traits principaux : le trait central oblique, le trait de gauche et le trait vertical de droite. Parfois le trait central oblique est prolongé par un sérif supplémentaire vers le haut. Contrairement au groupe sudouest, ici le trait vertical de gauche n'est presque jamais perpendiculaire à la ligne de base mais arrondi ; son extrémité inférieure est décalée vers la gauche par rapport au sommet du trait central, en donnant à la lettre un aspect ouvert en bas. Dans la plupart des cas, ce trait se termine par un tour de plume décoratif qui crée un court pied tourné vers la gauche, vers l'extérieur de la lettre. Le point de contact entre le trait vertical de gauche est le trait central oblique est à une certaine distance par rapport à l'extrémité supérieure de ce dernier. L'extrémité du trait central de l'aleph est placée à la hauteur de la ligne de crête. Le trait central oblique s'arrête souvent avant la ligne de crête mais est complété vers le haut par un sérif.

Pe est de la taille des lettres moyennes. Il est en principe composé de trois traits : la base presque parallèle à la ligne de base, le trait vertical de droite qui est légèrement penché ou arrondi vers la gauche, et le trait de droite qui touche au trait vertical de droite en créant un sommet pointu de la lettre et en descendant en ligne arrondie vers l'intérieur de la lettre. Contrairement au sous-type du Sud-Ouest, le pe n'a pas de barre horizontale supérieure. La partie haute de la lettre est formée par la rencontre des deux traits verticaux et leur mouvement - penché à droite et arrondi à gauche.

Cette brève comparaison de quelques traits pertinents des deux sous-types de l'écriture hébraïque orientale carrée résume les travaux menés au cours de cette année et constitue la base d'une étude détaillée d'un large corpus de manuscrits ne portant pas de date ni de mention de lieu de production, conservés à la Geniza du Caire.

Tableau 1. - Les caractéristiques principales des écritures carrées orientales

\begin{tabular}{|c|c|c|}
\hline Caractéristique & $\begin{array}{c}\text { Sud-Ouest } \\
\text { Exemple : MS TS 12.188 }\end{array}$ & $\begin{array}{c}\text { Nord-Est } \\
\text { Exemple : Codex Babylonicus } \\
\text { MS Firkovicz EBP I B 3 }\end{array}$ \\
\hline a & & \\
\hline $\mathrm{b}$ & &
\end{tabular}




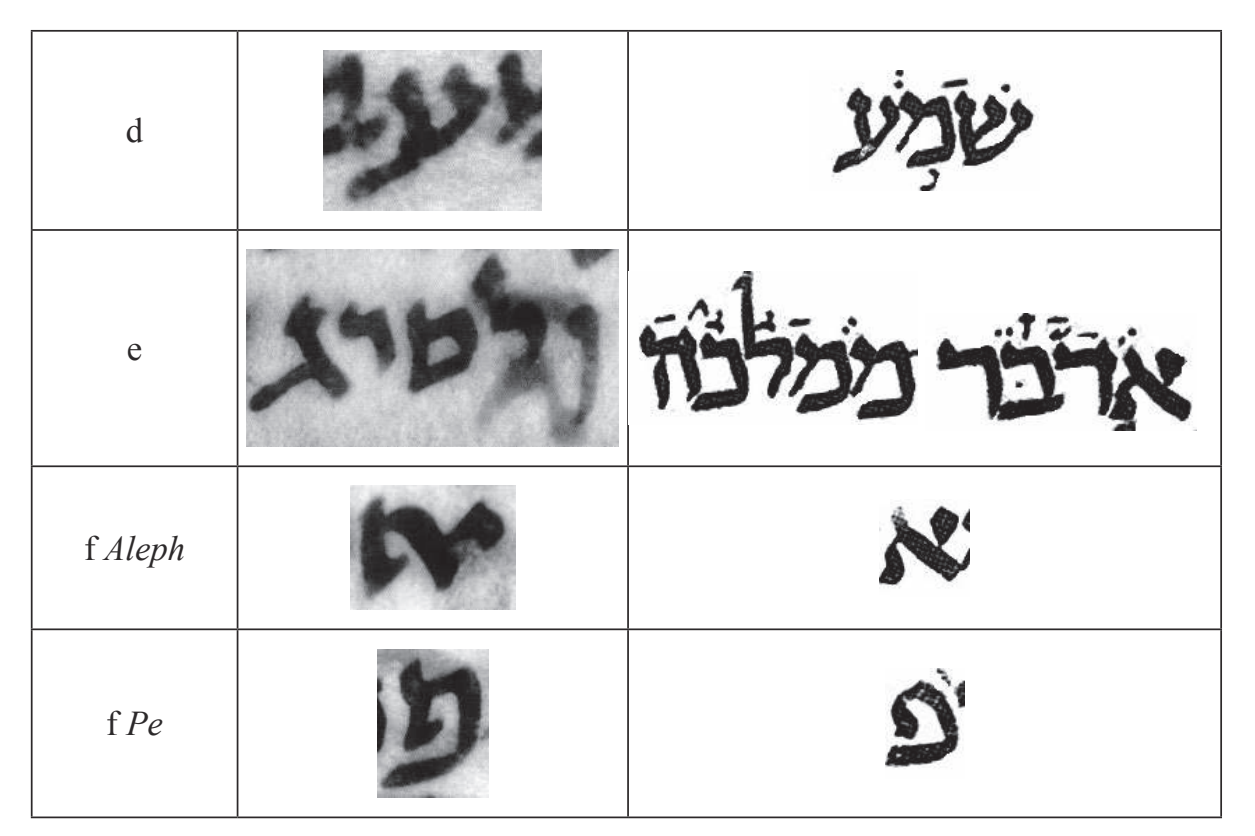

